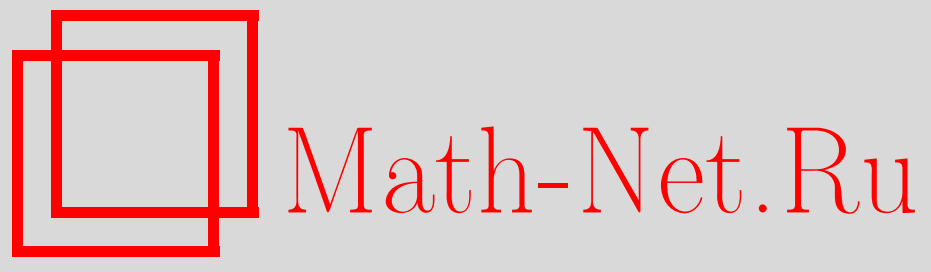

И. В. Закалюкин, Особенности управляемых систем при вырождении неголономных связей, УМН, 2010, том 65, выпуск 4, 193-194

DOI: https://doi.org/10.4213/rm9361

Использование Общероссийского математического портала Math-Net.Ru подразумевает, что вы прочитали и согласны с пользовательским соглашением http://www . mathnet.ru/rus/agreement

Параметры загрузки:

IP: 3.91 .87 .62

26 апреля 2023 г., 12:28:58

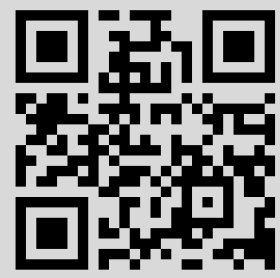




\section{Особенности управляемых систем при вырождении неголономных связей}

\section{И. В. Закалюкин}

В конфигурационном пространстве $M \approx \mathbb{R}^{n}$ с координатами $q$ рассмотрим управляемую систему $\dot{q}=u$, для которой управления $u \in T_{q} M$ будем считать принадлежащими распределению, заданному уравнениями

$$
A(q) u=0,
$$

где компоненты $a_{i j}(q)$ матрицы $A(q)$ размера $k \times n$ гладкие. Если ранг матрицы $A$ максимален, то множество $C \subset\{(q, \dot{q})\}$ решений системы (1) представляет собой гладкое подрасслоение $T M$. В общем положении для того, чтобы ранг матрицы $A$ упал на 1 , необходимо выполнение $n-k+1$ независимых условий (см. [1]), т. е. подмножество $\Sigma_{1} \subset M$ значений обобщенных координат, при которых ранг $A(q)$ равен $k-1$, является гладким подмногообразием коразмерности $n-k+1$. Пусть $\Sigma \subset C$ - это подмножество $T M \backslash\{(q, 0)\}$ точек фазового пространства, в которых матрица $A$ вырождена и имеет ранг $k-1$. Подмножество $\Sigma$ является гладким подмногообразием. Более того, множество $C$ допустимых связями фазовых положений остается неособым подмногообразием в окрестности $\Sigma$.

Теорема 1. Множество достижимости системы (1) общего положения для любой начальной точки содержит подмножество $\Sigma_{1}$, если выполнено неравенство $(n-k)(n-k+3)>n-2$.

Доказательство вытекает из теоремы Рашевского-Чжоу (см., например, [2]) и существования в общем положении допустимых обобщенных скоростей над всякой точкой из $\Sigma_{1}$, не касающихся $\Sigma$.

Рассмотрим теперь динамическую систему с кинематическими связями (1), заданную уравнениями Даламбера-Лагранжа с неопределенными множителями

$$
\frac{d}{d t} \frac{\partial T}{\partial \dot{q}_{i}}-\frac{\partial T}{\partial q_{i}}=Q_{i}+\sum_{j} \lambda_{j} \frac{\partial f_{j}}{\partial \dot{q}_{i}}, \quad A(q) \dot{q}=0, \quad i=1, \ldots, n, \quad j=1, \ldots, k,
$$

где $T=\frac{1}{2} \sum_{i, j=1}^{n} m_{i j}(q) \dot{q}_{i} \dot{q}_{j}$ - кинетическая энергия системы, являющаяся функцией обобщенных координат $q=\left(q_{1}, \ldots, q_{n}\right)$ и обобщенных скоростей $\dot{q}=\left(\dot{q}_{1}, \ldots, \dot{q}_{n}\right), Q_{i}-$ обобщенные силы, которые будем считать управлениями $U=\left(Q_{1}, \ldots, Q_{n}\right)$, не превосходящими по норме некоторой фиксированной константы, и $\lambda_{j}-$ неопределенные множители [3], [4].

Пусть $N=\left(n_{i j}\right)$ - матрица, обратная к матрице $\left(m_{i j}\right)$. В точках $\Sigma_{1}(k \times k)$-матрица $R=A N A^{*}$ ( $A^{*}$ - транспонированная к $A$ матрица) вырождена. С помощью замены базисных уравнений связей и локальной замены обобщенных координат матрицу $A$, имеющую в начале координат ранг $k-1$, можно привести к виду

$$
A=\left(\begin{array}{cc}
B & K \\
g_{1} \ldots g_{n-k+1} & 0 \ldots 0
\end{array}\right)
$$

где $(k-1) \times(k-1)$-минор $K=\left(a_{l m}\right)$ при $l=1, \ldots, k-1, m=n-k+2, \ldots, n$, отличен от нуля, а компоненты $g_{1}, \ldots, g_{n-k+1}$ - независимые функции координат, обращающиеся в нуль в начале координат.

Работа выполнена при поддержке РФФИ (грант № 08-01-157), программы "Ведущие научные школы" (грант НШ-709.2008.1) и гранта AVCP 2.2.1/5568. 
Обозначим через $G_{A} \in \mathbb{R}^{n}$ вектор с компонентами $g_{1}(q), \ldots, g_{n-k+1}(q), 0, \ldots, 0$, через $\Delta_{A}(q)$ определитель матрицы $R=A N A^{*}$ и через $\xi_{A}(q, \dot{q})$ квадратичную форму обобщенных скоростей $\xi_{A}(q, \dot{q})=\sum_{i=1}^{n} \sum_{j=1}^{n-k+1} \frac{\partial g_{j}}{\partial q_{i}} \dot{q}_{i} \dot{q}_{j}$.

Теорема 2. В некоторой окрестности $W$ начала координат $q_{0}$ существуют такие ограниченные вектор-функиии $F(q, \dot{q})$, что разрешенные относительно обобщенных ускорений уравнения Даламбера-Лагранжа в точках из $C$, проекиия которых на конфигурационное многообразие принадлежит $W \backslash \Sigma_{1}$, имеют вид:

$$
\ddot{q}=F(q, \dot{q})-\frac{\xi_{A}(q, \dot{q})}{\Delta_{A}(q)} N G_{A} .
$$

ЗАмечАниЕ 1 . Пусть $\|q\|_{A}$ - расстояние в некоторой метрике от точки $q$ до особого подмногообразия $\Sigma_{1}$. Функции $\Delta_{A}(q), D=\sum_{i=1}^{n-k+1} g_{i}^{2}$ и $\|q\|_{A}^{2}$ эквивалентны при $q \rightarrow \Sigma_{1}$. Вектор $N G_{A} / \Delta_{A}$, входящий в правую часть (4), имеет порядок $\|q\|_{A}^{-1}$.

ЗАмечание 2. Разложение (4) означает, что в окрестности $\Sigma$ уравнения Даламбера-Лагранжа представляют собой быстро-медленную систему: медленными переменными являются обобщенные координаты, а быстрыми - обобщенные скорости, производные которых задаются выражениями $\ddot{q}=P_{n}\|q\|_{A}^{-1}$, в которых компоненты вектор-функции $P_{n}(q, \dot{q})$ ограничены.

Теорема 3. Пусть $m=\left(q_{0}, \dot{q}_{0}\right) \in \Sigma_{1}$ - некоторал точка особого подмногообразия, в которой $\xi\left(q_{0}, \dot{q}_{0}\right) \neq 0 u \dot{q}_{0} \neq 0$. Тогда не существует ни одной непрерььно дифферениируемой фазовой траектории, трансверсально пересекающей подмногообразие $\Sigma_{1}$ в некоторый момент времени $t_{0}$ в точке $m$ и являющейся решением уравнений Даламбера-Лагранжа при некоторых равномерно ограниченных по норме обобщенных силах для $t \neq t_{0}$. Кроме того, для всякого $B \in \mathbb{R}$ существует открытое подмножество компактной области фазового пространства, содержащее точки из $\Sigma$ и не принадлежащее области достижимости для управляемой системы с обобщенными силами, ограниченными по норме константой $B$, и началъной точкой вне некоторой окрестности этого подмножества.

Примером такой системы являются уравнения плоского движения твердой балки, к концам которой шарнирно прикреплены два одинаковых конька, подчиняющихся стандартному "неголономному" условию: скорость в центре конька направлена вдоль его оси. В точках $\Sigma$ уравнения неголономных связей становятся зависимыми: оба конька становятся перпендикулярными стержню. Если к балке прикладывать произвольные управления в виде активных обобщенных сил, ограниченных сколь угодно большой константой, и начинать движение из произвольной точки фазового пространства вне $\Sigma$, то система никогда не придет в положение из $\Sigma$ с отличной от нуля квадратичной формой $\xi$.

\section{Список литературы}

[1] В.И. Арнольд, А.Н. Варченко, С. М. Гусейн-Заде, Особенности дифферениируемых отображений, 2-е изд., МЦНМО, М., 2004, 672 с. [2] А. А. Аграчев, Ю. Л. Сачков, Геометрическая теория управления, Физматлит, М., 2004, 391 с. [3] В. И. Арнольд, В. В. Козлов, А. И. Нейштадт, "Математические аспекты классической и небесной механики”, Итоги науки и техники. Современные проблемы матем. Фундам. напр., 3, ВИНИТИ, М., 1985, 5-290. [4] А. В. Борисов, И. С. Мамаев (ред.), Неголономные динамические системы. Интегрируемостъ, хаос, странные аттракторы, ИКИ, Ижевск, 2002, 328 с.

И. В. Закалюкин (I. V. Zakalyukin)

Московский авиационный институт

E-mail: zakalyukin@gmail.com
Представлено Ю. С. Ильяшенко Принято редколлегией 29.04 .2010 